\title{
CT-Guided Percutaneous Approach with Low-Dose Protocol for Performing Lung Biopsies
}

\author{
Gravina M*, Stoppino L, Loizzi D, Corallo F, \\ Punzi A, Garolla E, Manco MGR, Suriano S, \\ Quarta Colosso O, Ardò NP, Casavecchia G, Tango \\ S, De Bellis R, Sollitto F, Macarini $L$ and Vinci $R$ \\ Department of Medical \& Surgical Sciences, University of \\ Foggia, Italy \\ *Corresponding author: Matteo Gravina, Department \\ of Medical \& Surgical Sciences, University of Foggia, Viale \\ Pinto 1, 71122 Foggia, Italy
}

Received: March 26, 2021; Accepted: April 23, 2021; Published: April 30, 2021

\begin{abstract}
Computed tomography-guided percutaneous needle biopsy of the lung is a safe and effective procedure for removal of rustling of lung cancer in patients requiring histological diagnosis and to be able to carry out molecular biology studies, it does not require hospitalization and considerably limits the use of surgical biopsy. The purpose of our study is to assess the sensitivity and specificity of CT guided percutaneous procedure considering the best approach in order to obtain an acceptable number of withdrawals reducing patient exposure to ionizing radiation. Prior to the procedure we evaluate the best percutaneous approach studying the imaging features of the lung nodule. At the beginning of the procedure were performed a basic volumetric chest CT scan for the localization of the nodule to choose the point of access with bright laser pointer and successive CT scans of $3 \mathrm{~cm}$ thickness with a lowdose technique to follow the needle trajectory until the nodule of interest. Key point of this study is the use of 16-18 Gauge coaxial needle or introducer inch, to reach the target lesion; it allows multiple biopsies reducing the number of percutaneous accesses: in fact, reached the lesion, is possible to slide inside it the needle with semiautomatic shearing system of the Tru-Cut type and make multiple withdrawals. We performed 106 percutaneous CT-guided pulmonary needle biopsy on patients aged 41 to 89 years, 76 males and 30 females; in only 2 cases (1.88\% of the patients) we not obtained an accurate withdrawal of bioptic specimens for histological characterization of target lesion, and in just 2 cases the development of pneumothorax required placement of percutaneous thoracic drainage. This approach has been shown to have sensitivity and specificity of $98,1 \%$ and $97,1 \%$ respectively, for a proper selection of specimens and a correct histological diagnosis. A standardized and repeatable protocol for performing CT-guided biopsies is of key importance in order to reduce patient's exposure to ionizing radiation, to gain the highest possible number of appropriate withdrawals and to minimize procedural complications.
\end{abstract}

\section{Introduction}

In Italy lung cancer is the second most common cancer in men and the third in women, with about 42500 new cases estimated in 2019 [1]. For the histological characterization of lung cancer we have different possible techniques and the choice of the appropriate procedure is based on the type of tumor, its size, localization, and stage of disease (2). Computed tomography-guided percutaneous needle biopsy is the procedure used electively to study lung cancer, especially peripheral lesions, considering its high diagnostic sensitivity (about 90\%) [2] in patients where histological characterisation is required when radical surgery is not indicated. Pneumothorax is the most common complication, the incidence of which varies according to the size of needle used (rate of 10-30\%) and features of biopsied lesions [3]. This risk increases especially when it is necessary to sample small lesions ( $<2 \mathrm{~cm}$ in diameter), sub-solid or ground-glass lesions, central endobronchial and/or non-adhesive to the chest wall nodular lesions [4,5]. Before performing a CT guided biopsy, it is a good practice to make a preliminary evaluation of shape, size, and localization of lung nodule and carefully select the size of the needle to use [6]. The purpose of our study is to evaluate how to get the best possible percutaneous access and biopsy with an appropriate number of specimens, minimizing the complications procedure related. In our work we adopted a standardized protocol, repeatable by all operators and optimised to minimize patient exposure to ionizing radiation. Another key element of our study is the choice to always use an introductory (or coaxial) needle to reach the lesion to be biopsied and to follow its trajectory in the chest wall by targeted CT scans. The use of introductory needle allows us to perform multiple biopsy, reducing the number of percutaneous accesses: after reaching the lesion with needle, removed the core of the needle flows inside it the cutting needle of the Tru-Cut device. The aim of our study was to carry out a retrospective analysis of CT-guided lung biopsies performed at "Radiology Department - Foggia University" from January 2017 to January 2020, evaluating the success of the procedure in terms of taking an accurate amount of biopsy specimens and its possible related complications.

\section{Materials and Methods}

The technique we used for CT guided percutaneous histological sampling consists in the insertion of an introductory centimetred needle, whose gauge is appropriate to the nodule's size that will be biopsied (16 and 18 gauge needles). As we reach the lesion, we put inside the needle's cannula the semiautomatic system of the 
Tru-cut and through it we perform multiple withdrawals. Before performing the guided CT biopsy, should be analysed the shape, size and localization of the lung's nodule with a chest CT scan without contrast and with normal breathing $[7,8]$. Trought this scan is also possible to assess the presence of pleural effusion, consolidation of the parenchyma or other pathological conditions that could affect the success of the procedure (assessment of surgical planning). The next step involves choosing the decubitus of the patient (supine, prone or lateral) and evaluating the best access for the introductory needle. The percutaneous route of the coaxial needle should be as short as possible, avoid skeletal structures (especially ribs, vertebral and sternum), heart and major mediastinal vascular structures.

The patient is placed in the chosen decubitus and is performed a CT scan of the considered region. Trought the obtained CT scan we evaluate two variables in order to choose a needle of suitable length: the angle of inclination that will be used to insert the coaxial needle and the distance between the point of access on the skin and the target lesion. At this point, the light center of the gantry is used to identify, on the patient's chest, the point corresponding to the chosen way of access. After skin disinfection, local anesthesia is performed with 5-10 cc of Lidocaine and a perforated sterile cloth is placed. The needle used for the anesthetic injection can be used in a second CT scan as a radiopaque marker in order to assess the precision of the position of both the access point and the trajectory given to the biopsy needle. Afterwards the coaxial needle is introduced and its position during the progression is controlled by one or two CT scans until the lesion is reached, changing the scanning parameters in order to minimize the radiant dose (usually scans with $80 \mathrm{mV}$ and $70 \mathrm{~mA}$ ). If a large-caliber introductory needle is used, a hole is opened on the skin using a scalpel. After reaching the lesion with the introductory needle, we can put inside the Tru-Cut cutting needle trought, which take multiple biopsies, on average four. At the end of the biopsy, when the needle is removed, a low dose chest CT scan is always performed to exclude the presence of post-procedural complications. The patient will be kept for an observation of 6-12 hours in his provenance department; he will be discharged after a clinical evaluation and a chest X-Ray examination in two projections performed 6 hours after the procedure. In this study we evaluated several parameters likely to be related with the success of the procedure reducing the complications:

- Length of aerated lung parenchyma crossed by the biopsy needle.

- $\quad$ Needle gauge.

- The single passage through the pleural surface.

- The position of the patient.

The success of the examination, is related both to the collection of an appropriate amount of biopsy rustles (to be subjected to the anathomo-pathological study) and to the careful clinical evaluation of patients applying for the procedure, in order to avoid complications such as bleeding because of the previous suspension of anti-aggregating/anticoagulant therapies or the correction of coagulopathies (5). Before the execution of the lung biopsy, informed consent has been obtained from all patients after an accurate information about the examination technique and the possible risks. Patients with a INR $>1.4$ or platelet count $<50000 / \mu$ l have been

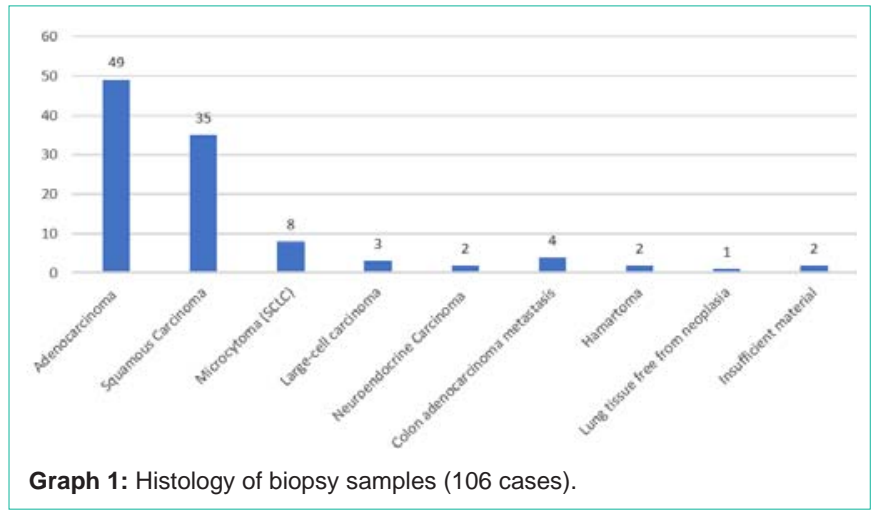

excluded from the study. Prophylactic administration of antibiotics is not routinely performed, as the procedure is performed in sterility conditions and therefore with substantially low risk of infection.

In our center were performed $106 \mathrm{CT}$-guided percutaneous lung agobiopsy, on 86 males and 20 women, aged between 46 and 87 years, at the Complex Unit of University Radiodiagnostics of the United Hospitals of Foggia, in the period from January 2017 to January 2020. All procedures were performed by the same operator.

In our case studies the most common malignant histological type was adenocarcinoma with 49 cases out of 106 corresponding to 46.2\%; squamous carcinoma in 35 cases (33\%), Small Cell Carcinoma (SCLC) or microcytoma in 8 cases (7.54\%), large cell lung carcinoma in 3 cases (2.83\%), 2 cases of neuroendocrine carcinoma in large cells (1.88\%) (Graph 1). In addition, 4 cases (3.77\%) of secondary lung locations from adenocarcinoma of the large intestine were found, in 2 cases $(1.88 \%)$ the tissue taken was found to belong to benign nodules, both amartoms, 1 case $(0.9 \%)$ the tissue taken was free from malignant neoplasm while in 2 cases $(1.88 \%)$ the material taken was judged inadequate or insufficient for a proper anatomy-pathological evaluation.

In our study there are 50 patients $(47.17 \%)$ presenting lesions attached to the chest wall or with normal aerated pulmonary tissue interposition between lesion and pleura lesser than $1 \mathrm{~cm}$; in remaining 56 patients $(52.83 \%)$ we have intra-parenchymal lesion with normal aerated pulmonary tissue interposition between lesion and pleura ranging from $11 \mathrm{~mm}$ to $55 \mathrm{~mm}$ (medium distance $24 \mathrm{~mm}$ ).

In the first group we had 2 cases $(1.88 \%)$ of pneumothorax and 1 case $(0.94 \%)$ of pleural blood effusion while in the second group there were 6 cases $(5.66 \%)$ of pneumothorax and 2 cases $(1.88 \%)$ of pleural blood effusion; therefore we had a total of 8 cases $(7.54 \%)$ of pneumothorax and 3 cases $(2.83 \%)$ of pleural blood effusion.

Intraprocedurally pulmonary haemorrhage we caused was mild, so there was no need for transfusions or invasive procedures to stop bleeding.

We need for chest tube insertion in only 2 cases because the air collection into the pleural cavity was significant with massive pulmonary collapse; in the other 6 cases pneumothorax was not significant and we use the coaxial biopsy needle to aspirate the air from the pleural space prior to removal of the needle.

In our study, lesion depth greater than $20 \mathrm{~mm}$, with normal 
aerated pulmonary tissue interposition between lesion and pleura, and smaller lesion size $<1.5 \mathrm{~cm}$ was determined to be a significant risk factor for pneumothorax.

The paraseptal and centrilobular pulmonary emphysema as well as pulmonary fibrosis does not seem to affect the incidence of pneumothorax or other complications.

In our study lesions sizes ranged from $18 \mathrm{~mm}$ to $105 \mathrm{~mm}$, with an average size of $32 \mathrm{~mm}$; pneumothorax occurred in nodules between 18 and $34 \mathrm{~mm}$ in size.

In only 2 cases we did not obtain an appropriate amount of tissue sample for diagnosis; nodules, in these two cases, had a distance from the chest wall varying from 51 to $76 \mathrm{~mm}$.

Although we had a few cases of failure in order to obtaining an appropriate amount of samples, we can consider that this result is related to the small size of the lesions, the distance from the chest wall and the size of the needle $[9,10]$.

\section{Discussion}

CT-guided percutaneous lung biopsy is a useful diagnostic procedure for the study of suspected pulmonary malignancy in patients whit no indications for surgery, who needs an histological diagnosis [11].

In this study we investigated the best percutaneous approach in order to obtain an appropriate number of biopsy samples using an optimized technique and reducing patient's exposure to ionizing radiation [12].

The patients were placed in the supine, prone, or lateral position according to the location of the lesion. A basic non-contrast CT scan was performed to correctly locate the target lesion; after selecting the skin entry point of the biopsy needle using positioning laser lights of the gantry, two volumetric scans of $3 \mathrm{~cm}$ thick with low-dose protocol ( $80 \mathrm{kV}$ and $80 \mathrm{~mA}$ ) were performed to follow the needle path to the lesion. Performing the procedure with low-dose protocol we obtained a decrease in the radiant dose of about $50 \%$.

In order to obtain a standardised procedure, it is necessary to evaluate the anatomical variants between the subjects and the equipment. To obtain a protocol that is in accordance with anatomical variants we should take into account the antero-posterior AP and latero-lateral LL dimensions of each patient using a Size Specific Dose

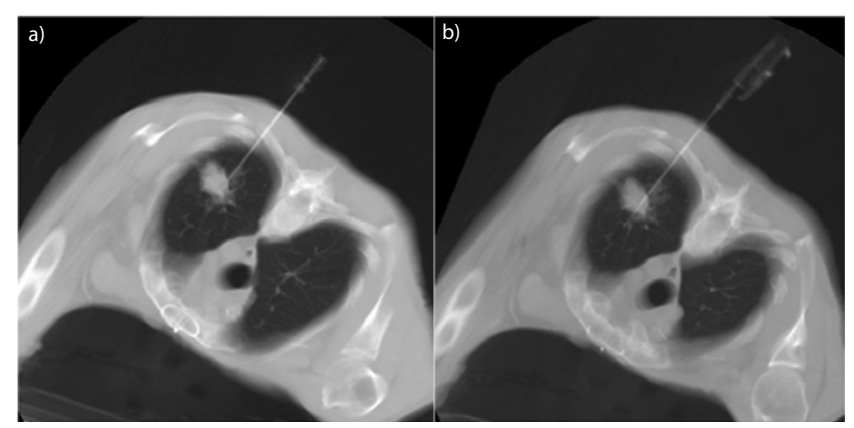

Figure 1: CT guided percutaneous needle biopsy: a) positioning of coaxial introducer needle, b) insertion of a semiautomatic tru-cut guillotine needle inside it.

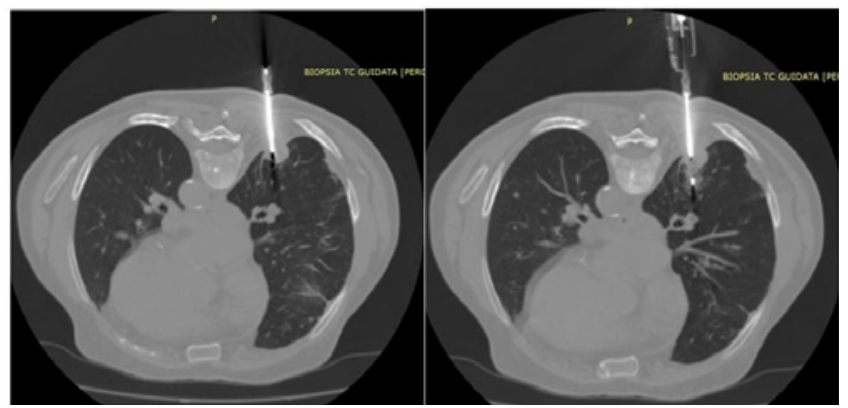

Figure 2: CT-guided percutaneous needle biopsy of nodular formation of the posterior segment of the lower lobe of the right lung after insertion of a coaxial introducer needle in a patient in prone decubitus.

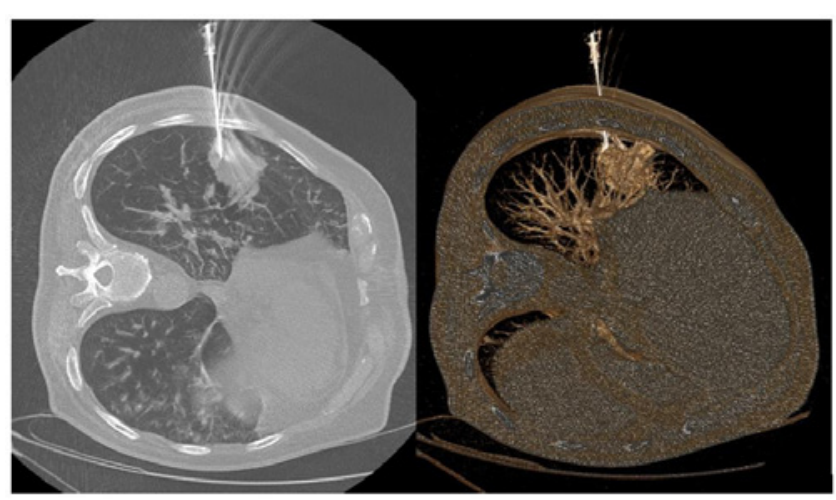

Figure 3: Pulmonary needle biopsy of the lateral segment of the lower lobe of the right lung with the patient in left lateral decubitus.

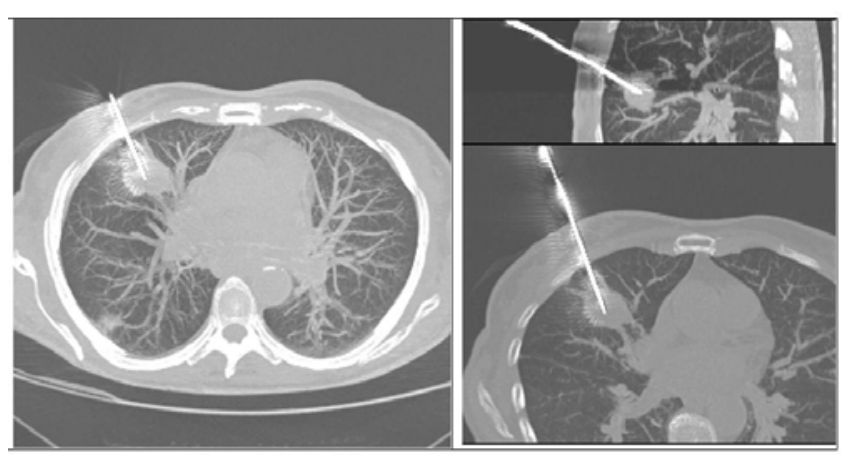

Figure 4: CT-guided needle biopsy of the anterior segment of the upper lobe of the right lung.

Estimation (SSDE) of $80 \mathrm{mGy}$ as reference parameters.

The key point of our study is the use of 16 or 18Gauge coaxial introducer needle to reach the lesion; this device allows to obtain multiple biopsy samples minimizing pleural transgression by performing a single pleural puncture and avoiding fissures if possible $[13,14]$.

After reaching the lesion the internal stylet of the introducer needle is replaced by the Tru-Cut type cutting needle. The Tru-Cut type cutting needle consists of an outer cutting cannula and an inner slotted stylet (Figure 1). After insertion of the introducer needle tip to the edge of the lesion, the inner slotted stylet is advanced and 
followed by the outer cutting cannula, which slices a piece of tissue.

In our procedure the coaxial introducer needle is held in a fixed position and the Tru-Cut needle is inserted several times in order to take multiple tissue samples; with larger lesions, it is possible to make minimal excursions of the coaxial introducer needle obtaining specimens from a larger area of the lesion (Figure 2).

This technique reduces trauma through the chest wall because it is based on single access for multiple histological samples (Figure 3). Specimen acquisition was repeated until the operator thought that the specimens were adequate. Core biopsy specimens should be placed directly in formalin for fixation. The mean number of specimens obtained per lesion was 4 .

Moreover, in this way we can minimize the seeding of neoplastic cells along the path of the needle because the Tru-Cut needle passes through the coaxial needle cannula and has no contact with the chest wall [15] (Figure 4).

If a pneumothorax occurs during or following a lung biopsy procedure, the air should be aspirated from the pleural space using the same coaxial needle: the distal end of the coaxial needle is placed in the pleural cavity and the proximal end (outside the chest) is connected to a three-way stopcock with connexion tubing; the air can be aspirated with a $50 \mathrm{cc}$ Luer-Lock syringe [16]. This minimizes the need for chest tube insertion: we used this procedure in only two cases in our study [17-19].

\section{Conclusion}

Needle biopsy is a minimally invasive technique of tissue sampling that plays a role in obtaining pathologic proof of malignancy, guiding staging and planning treatment [20]. A standardized and repeatable protocol for CT-guided biopsies allows not only to minimize patients' exposure to ionizing radiation but also represents an effective strategy in order to obtain tissue for the diagnosis of indeterminate pulmonary lesions, minimizing procedure complications.

Compared to excisional or open biopsy, percutaneous CT-guided needle biopsy can be performed in most cases on a day-surgery or on an outpatient basis.

If carried out by qualified medical staff, it is a safe and efficient procedure that exposes patients to few risks and allows personalized oncological treatment, assuming an important role for patients therapeutic plan.

\section{References}

1. I Numeri del Cancro in Italia 2019 - AION.

2. Rivera MP, Mehta AC, Wahidi MM. "Establishing the Diagnosis of Lung Cancer." Chest. 2013; 143: e142S-e165S.

3. Choi SH, Chae EJ, Kim JE, et al. "Percutaneous CT-guided aspiration and core biopsy of pulmonary nodules smaller." AJR Am J Roentgenol. 2013; 201: 964-970.

4. Veltri A, Bargellini I, Giorgi L, Almeida PAMS, Akhan O. "Cardiovascular and Interventional Radiological Society of Europe: Guidelines on Percutaneous Needle Biopsy (PNB)" Cardiovasc Intervent Radiol. 2017.
5. Tam AL, Lim HJ, Wistuba II, Tamrazi A, Kuo MD, Ziv E, et al. "Image-guided biopsy in the era of personalized cancer care: proceedings from the society of interventional radiology research consensus panel." J VascInterv Radiol. 2016; 27: 8-19.

6. ACR-SIR-SPR. "Practice parameter for the performance of image-guided percutaneous needle biopsy (PNB)" Res. 35-2013, Amended 2014 (Res. 39).

7. Tam AL, Kim ES, Lee JJ, Ensor JE, Hicks ME, Tang X, et al. "Feasibility of image-guided transthoracic core-needle biopsy in the BATTLE lung trial." J Thorac Oncol. 2013; 8: 436-442.

8. Gupta S. "New techniques in image-guided percutaneous biopsy." Cardiovasc Intervent Radiol. 2004; 27: 91-104

9. Chehab MA, Brinjikji W, Copelan A, Venkatesan AM. "Navigational tools for interventional radiology and interventional oncology applications." SeminInterventRadiol. 2015; 32: 416-427.

10. Aviram G, Greif J, Man A, Schwarz Y, Marmor S, Graif M, et al. "Diagnosis of intrathoracic lesions: are sequential Fine-Needle Aspiration (FNA) and Core Needle Biopsy (CNB) combined better than either investigation alone?" ClinRadiol. 2007; 62: 221-226.

11. Prosch H, Stadler A, Schilling M, Bu r̈klin S, Eisenhuber E, Schober E, et al "CT fluoroscopy-guided vs. multislice CT biopsy mode-guided lung biopsies: accuracy, complications and radiation dose." Eur J Radiol. 2012; 81: 10291033.

12. Mammarappallil JG, Hiatt KD, Ge Q, Clark HP. "Computed tomography fluoroscopy versus conventional computed tomography guidance for biopsy of intrathoracic lesions: a retrospective review of 1143 consecutive procedures." J Thoraclmaging. 2014; 29: 340-343.

13. Gupta S, Wallace MJ, Cardella JF, Kundu S, Miller DL, Rose SC. "Society of Interventional Radiology Standards of Practice Committee. Quality improvement guidelines for percutaneous needle biopsy." J Vasc Interv Radiol. 2010; 21: 969-975.

14. Gupta S, Seaberg K, Wallace MJ, Madoff DC, Morello FA, Ahrar K, et al. "Imaging-guided percutaneous biopsy of mediastinal lesions: different approaches and anatomic considerations." Radiographics. 2005; 25: 763788.

15. Agarwal P, Seely J, Matzinger F, et al. "Pleural mesothelioma: sensitivity and incidence of needle tract seeding after image-guided biopsy versus surgical biopsy." Radiology. 2006; 241: 589-594.

16. Tomiyama N, Yoshifumi Y, Yasuo N, et al. "CT-guided needle biopsy of lung lesions: a survey of severe complications based on 9783 biopsies in Japan." Eur J Radiol. 2006; 59: 60-64.

17. Moreland A, Novogrodsky E, Brody L, Durack J, Erinjeri J, Getrajdman G, et al. "Pneumothorax with prolonged chest tube requirement after CT-guided percutaneous lung biopsy: incidence and risk factors." EurRadiol. 2016; 26 : 3483-3491.

18. Heerink WJ, de Bock GH, de Jonge GJ, Groen HJ, Vliegenthart R, Oudkerk M. "Complication rates of CT-guided transthoracic lung biopsy: metaanalysis." EurRadiol. 2017; 27: 138-148.

19. Atwell TD, Smith RL, Hesley GK, Callstrom MR, Schleck CD, Harmsen WS, et al. "Incidence of bleeding after 15,181 percutaneous biopsies and the role of aspirin." Am J Roentgenol. 2010; 194: 784-789.

20. Pua BB, Solomon SB. Lymph Node Biopsy. Editors. In: Gervais DA, Sabharwal T. "Interventional radiology procedures in biopsy and drainage." London: Springer. 2011; 73-79. 